\title{
VoIP over WLAN: Voice capacity, admission control, QoS, and MAC
}

\author{
Lin Cai ${ }^{1, \$}$, Yang $\mathrm{Xiao}^{2, \S}$, Xuemin (Sherman) Shen ${ }^{1, *, \dagger}$, Lin $\mathrm{Cai}^{3, \uparrow}$ \\ and Jon W. Mark ${ }^{1,}$ \\ ${ }^{1}$ Centre for Wireless Communications in the Department of Electrical and Computer Engineering, \\ University of Waterloo, Waterloo, Ont., Canada N2L $3 G 1$ \\ ${ }^{2}$ Department of Computer Science, University of Memphis, U.S.A. \\ ${ }^{3}$ Department of Electrical and Computer Engineering, University of Victoria, Canada
}

\begin{abstract}
SUMMARY
Voice over Internet Protocol (VoIP) is one of the fastest growing Internet applications. It is a viable alternative to the traditional telephony systems due to its high resource utilization and cost efficiency. Meanwhile, Wireless Local Area Networks (WLANs) have become a ubiquitous networking technology that has been deployed around the world. Driven by these two popular technologies, Voice over WLAN (VoWLAN) has been emerging as an infrastructure to provide low-cost wireless voice services. However, VoWLAN poses significant challenges since the performance characteristics of wireless networks are much worse than that of their wireline counterparts, and the IEEE 802.11-based WLAN was not originally designed to support delay-sensitive voice traffic. In this paper, we provide a survey of recent advances in VoWLAN voice capacity analysis, call admission schemes, and medium access control (MAC) layer quality of service (QoS) enhancement mechanisms. Some open research issues are presented for further investigation. Copyright (C) 2006 John Wiley \& Sons, Ltd.
\end{abstract}

KEY WORDS: VoIP; WLAN; network capacity; admission control; QoS; MAC

\section{INTRODUCTION}

Voice over Internet Protocol (VoIP), also known as IP telephony or Internet telephony, is a set of protocols to transport voice traffic over IP-based packet-switched networks with acceptable quality of service (QoS) and reasonable cost. Efforts in transmitting voice over packet-switched networks can be traced back to the early 1970s. Since the mid 1990s, IP telephony service has advanced rapidly, and it is anticipated as a viable alternative to the traditional voice service over

\footnotetext{
*Correspondence to: X. Shen, Department of Electrical and Computer Engineering, University of Waterloo, Waterloo, Ont., Canada N2L 3G1.

†E-mail: xshen@bbcr.uwaterloo.ca

E-mail: lcai@bbcr.uwaterloo.ca

${ }^{\S}$ E-mail: yangxiao@ieee.org

EE-mail: cai@uvic.ca

"E-mail: jwmark@bbcr.uwaterloo.ca
} 
public switched telephone networks (PSTN) due to its cost effectiveness. In addition, VoIP can efficiently provide compelling features and services, such as voice mail, voice conferencing, etc., by allowing the integrated transmission of voice and data over the same network. On the other hand, the Wireless Local Area Network (WLAN) becomes popular to support high-data-rate Internet access for users in proximity of an access point (AP). The main advantages of WLAN are its simplicity, flexibility and cost effectiveness. In the past several years, the IEEE 802.11 WLAN has become a ubiquitous networking technology and has been widely deployed around the world. Although most existing WLAN applications are data centric, such as web browsing, file transfer and electronic mail, there is a growing demand for multimedia services over WLANs.

Recently, VoIP over WLAN (Voice over WLAN, VoWLAN) has been emerging as an infrastructure to provide wireless voice service with cost efficiency. Driven by the demand from education, health care, retail, logistics, etc., VoWLAN will experience a dramatic increase in the near future. However, supporting voice traffic over WLANs poses significant challenges since the performance characteristics of the physical and MAC layers are much worse than that of their wireline counterparts. Therefore, the applications of VoWLAN raise several deployment issues concerning the system architecture, network capacity and admission control, QoS provisioning, etc.

In this paper, we first briefly overview the VoIP system and legacy IEEE 802.11 MAC protocol in Section 2. We then present a comprehensive survey on the voice capacity analysis and call admission control in Section 3. Recent advances of QoS enhancement schemes in the MAC layer are summarized in Section 4. Section 5 discusses the emerging vertical handoff and admission control issues for VoWLAN/cellular systems, followed by concluding remarks and open issues in Section 6.

\section{VoIP AND IEEE $802.11 \mathrm{MAC}$}

\subsection{VoIP system}

We focus on the packet-level VoIP system performance. There are three indispensable VoIP components at the end-systems: codecs, packetizer and playout buffer, as shown in Figure 1. The analogue voice signals are digitized, compressed and then encoded into digital voice streams

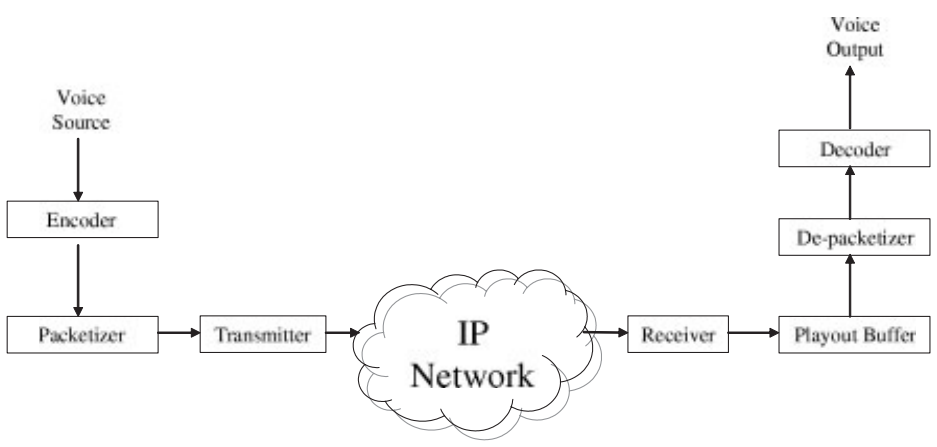

Figure 1. VoIP system. 
Table I. Attributes of commonly used codecs.

\begin{tabular}{lcccccc}
\hline Codec & $\begin{array}{c}\text { Bit Rate } \\
(\mathrm{kbps})\end{array}$ & $\begin{array}{c}\text { Sample Period } \\
(\mathrm{ms})\end{array}$ & $\begin{array}{c}\text { Compression } \\
\text { Ratio }\end{array}$ & $\begin{array}{c}\text { Payload } \\
\text { (bytes) }\end{array}$ & Packet/s & MOS** \\
\hline G.711 & 64 & 20 & $2: 1$ & 160 & 50 & 4.1 \\
G.723.1a & 5.3 & 30 & $8: 1$ & 20 & 33 & 3.65 \\
G.723.1a & 6.3 & 30 & $7: 1$ & 24 & 33 & 3.9 \\
G.729 & 8 & 10 & $8: 1$ & 10 & $50^{*}$ & 3.7 \\
\hline
\end{tabular}

*For G.729, two frames are combined into one packet; **MOS: mean opinion score.

\begin{tabular}{|c|c|c|c|c|}
\hline \multirow{3}{*}{$\begin{array}{l}\text { Application Layer } \\
\text { Transport Layer }\end{array}$} & \multicolumn{4}{|c|}{ Voice } \\
\hline & RTP & RTCP & SIP & H.323 \\
\hline & \multicolumn{4}{|c|}{ UDP } \\
\hline Network Layer & \multicolumn{4}{|c|}{ IP } \\
\hline Link Layer & \multicolumn{4}{|c|}{$802.3 / 802.11$} \\
\hline Physical Layer & \multicolumn{4}{|c|}{ Ethernet/SDH/RF } \\
\hline
\end{tabular}

Figure 2. VoIP protocol stack.

by the codec. Voice codecs are standardized by the International Telecommunication Union-Telecommunication (ITU-T), such as G.711 with the data rate of $64 \mathrm{kbps}$, G.729 with $8 \mathrm{kbps}$, G.723.1a with $5.3 / 6.3 \mathrm{kbps}$, etc. The main attributes of some commonly used encoding schemes are listed in Table I. The output voice stream then enters the packetizer to generate constant bit rate (CBR) audio packets with RTP(RTCP)/UDP/IP header where RTP and RTCP [1] are Real-time Transport Protocol and Real-Time Control Protocol, respectively, which are designed to support real-time multimedia applications with stringent delay constraint over unreliable User Datagram Protocol (UDP). Besides these, call set-up signalling protocols, such as Session Initiation Protocol (SIP) and H.323, are used for establishing VoIP connections. SIP, defined in RFC 2543 of Internet engineering task force (IETF), is a signalling protocol for Internet applications, e.g. conferencing, telephony, events notification, and instant messaging. H.323 standardized by ITU-T has been especially focused on smooth interworking with the PSTN.

The VoIP protocol stack is illustrated in Figure 2. Voice packets are transmitted over the IP network, and the reverse processes of decoding and depacketizing are accomplished at the receiver. A playout buffer is used by the receiver to smoothen the speech by getting rid of delay jitter. Packets arriving later than the playout time will simply be discarded. Some other components such as voice/silence detector, loss/error concealment and echo canceller, are also included in the system to enhance the functionality and performance of VoIP systems. The major metric to evaluate the user-perceived voice quality is the Mean Opinion Score (MOS), ${ }^{* *}$ which is rated on a scale of $1-5$.

\footnotetext{
**To determine MOS, a number of listeners rate the quality of test sentences transmitted over the communication system as follows: (1) bad; (2) poor; (3) fair; (4) good; and (5) excellent. The MOS is the arithmetic mean of all the individual scores.
} 


\subsection{IEEE 802.11 MAC}

IEEE 802.11 standard supports WLAN in two different modes, infrastructure and ad hoc. In the infrastructure mode, mobile nodes communicate with each other via an AP, while in the ad hoc mode, nodes communicate in a peer-to-peer fashion. VoWLAN applications mainly run over the infrastructure-based WLANs. There is a variety of standards defined in the IEEE 802.11 family: the $802.11 \mathrm{a}$ operates in the $5 \mathrm{GHz}$ frequency band and can provide up to $54 \mathrm{Mbps}$ raw data rate; the $802.11 \mathrm{~b}$ can provide up to $11 \mathrm{Mbps}$ raw data rate in the $2.4 \mathrm{GHz}$ frequency band; the $802.11 \mathrm{~g}$ operates in the same band as that of $802.11 \mathrm{~b}$, but can provide raw data rate up to $54 \mathrm{Mbps}$ by using the Orthogonal Frequency Division Multiplexing (OFDM) technology. Currently, $802.11 \mathrm{~b}$ is the most widely deployed standard worldwide, whereas $802.11 \mathrm{~g}$ is receiving acceptance because of the high rate and backward compatibility with $802.11 \mathrm{~b}$.

The IEEE 802.11 MAC defines two functions, the mandatory distributed co-ordination function (DCF) and the optional point co-ordination function (PCF) [2]. DCF is based on the carrier sense multiple access with collision avoidance (CSMA/CA) mechanism. All stations with frames ready for transmission monitor the shared medium before attempting to transmit. If the medium is sensed busy, the station defers transmission until the medium is sensed idle for a period of time equal to a DCF InterFrame Space (DIFS). After a DIFS medium idle time, the station enters the backoff phase in which it sets a random backoff counter randomly chosen from $[0, \mathrm{CW}]$ where $\mathrm{CW}$ is the current contention window $(\mathrm{CW})$, and $(\mathrm{CW}+1)$ is the contention window size. The backoff counter decreases by one for every idle slot and freezes if the channel is busy. In the latter case, the decrement procedure resumes after the channel is sensed idle again for a DIFS. The station transmits the frame when the backoff counter reaches zero. If there is no acknowledgment (ACK) received due to collision or transmission errors, the contention window size, i.e. $(\mathrm{CW}+1)$, doubles until it reaches the maximum value, and the sender reschedules the transmission according to the aforementioned backoff rule. Following every successful transmission, $\mathrm{CW}$ is reset to its initial (minimal) value. The frame is dropped when the retransmission limit is reached. If a frame is received successfully, the receiver transmits an ACK following a Short InterFrame Space (SIFS).

DCF was designed mainly for asynchronous data transmission, without the consideration of delay-sensitive real-time applications. An optional PCF is proposed to provide certain level of QoS based on a polling scheme; during the contention-free period (CFP), the point co-ordinator (PC), usually residing in the AP, maintains a polling list determining the sequence to poll stations.

\section{VOICE CAPACITY AND ADMISSION CONTROL}

\subsection{Voice capacity of DCF-based WLAN}

When designing a VoWLAN system, one of the most important issues is to determine the network voice capacity, in terms of the maximum number of simultaneous voice connections that can be supported in an IEEE 802.11 WLAN, especially for the prominently deployed DCF-based WLANs.

The performance of DCF-based WLANs has been extensively studied in the past decade. It has been shown in Reference [3] that due to the overhead, a theoretical throughput upper bound and delay lower bound exist for the DCF-based WLAN even if the transmission 
rate of mobile stations goes into infinite. Thus, reducing overhead is vital. In the literature, many existing throughput and delay analyses are based on the model that the active mobile stations are saturated, i.e. they always have data to transmit [4]. However, the saturatedstation model is not suitable for voice traffic, which is usually considered as a constant bit rate traffic, or as a variable bit rate traffic if considering the alternating periods of talk spurts and silence [5].

Voice capacity of WLANs has been actively investigated via experiments and analytical models. In Reference [6], the performance and capacity of the legacy IEEE 802.11b DCF-based WLAN delivering voice traffic has been measured, using a testbed consisting of commercially available components. It shows that the $802.11 \mathrm{~b}$ can support ten voice connections using voice codec G.711, a $10 \mathrm{~ms}$ packetization interval, and silence suppression. A measurement experiment with the voice codec G.711 without silence suppression has been carried out in Reference [7], which indicated that only six VoIP connections can be accommodated in an 802.11 b WLAN. Chariot, a widely used commercial network analysis tool, was used to estimate the voice capacity in Reference [8] that the 802.11 b can support eight to ten G.711 voice connections with $20 \mathrm{~ms}$ packetization interval and fourteen to eighteen G.723.1 voice connections with $30 \mathrm{~ms}$ packetization interval. The different measurement results show that the silence suppression and packetization interval play an important role on WLAN voice capacity.

On the other hand, analytical models have been proposed in References [9-11] to evaluate the voice capacity of infrastructure, DCF-based WLANs. The analytical results further quantify how the system parameters affect the WLAN voice capacity. The analytical model in Reference [9] considered the header overhead of each layer (e.g. RTP, UDP, IP, MAC and physical layer headers) and the overhead introduced by the MAC protocol, including DIFS, SIFS, ACK, and the random backoff, and simplified the analysis with the assumptions that there are no collisions and all mobile stations take advantage of the backoff time of the AP to fulfil their own backoff requirements. An improved analytical model has been proposed in Reference [10] and a tighter upper bound of voice connections in an $802.11 \mathrm{a} / \mathrm{b}$ WLAN has been obtained by assuming that there are always two active stations competing for the wireless channel.

A more accurate model has been developed in Reference [11] by considering the details of the CSMA/CA mechanism and the practical AP-bottleneck effect induced by the imbalanced traffic of the AP and mobile stations. Since all traffic to and from the WLAN has to go through the $\mathrm{AP}$, the traffic arrival rate of the AP is the summation of that of all mobile stations. When more voice connections join in the network, the service rates of the AP and mobile stations decrease non-linearly because more collisions may occur. A station is considered unstable when its traffic arrival rate is larger than the frame service rate, and the queue of the station will build up, and thus the voice connection will suffer from excessive delay and packet losses due to buffer overflows. It is pointed out that when the number of voice connections approaches the voice capacity, one more VoIP connection may cause the AP to be unstable, and thus jeopardize the performance of all downlink voice traffic.

The analytical results of the voice capacity with different codecs and packetization intervals are compared in Table II. The results in Reference [11] are tighter than those in References $[9,10]$ and match well with the simulation and measurement results, because of the accurate modelling of the CSMA/CA mechanism and collision events.

All of the above analytical models assume a constant transmission rate in the WLAN. For the adaptive transmission rate case, the voice capacity of an IEEE 802.11a WLAN was estimated in Reference [12]. 
Table II. Comparison of the maximum number of VoIP connections (802.11b).

\begin{tabular}{lrrrrrrrrrr}
\hline Packet & & G.711 & \multicolumn{3}{c}{ G.729 } & & G.723 & iLBC* \\
interval (ms) & {$[11]$} & {$[10]$} & {$[9]$} & {$[11]$} & {$[10]$} & {$[9]$} & {$[11]$} & {$[10]$} & {$[9]$} & {$[11]$} \\
\hline 10 & 6 & 6 & 6 & 6 & 7 & 7 & & & & \\
20 & 11 & 12 & 12 & 13 & 14 & 14 & & & & 12 \\
30 & 15 & 17 & 18 & 19 & 21 & 22 & 19 & 21 & 22 & 18 \\
40 & 19 & 21 & 22 & 25 & 28 & 28 & & & & \\
50 & 22 & 25 & 26 & 31 & 34 & 35 & & & \\
60 & 25 & 28 & 29 & 37 & 41 & 42 & 37 & 42 & 42 & \\
\hline
\end{tabular}

*Internet low-bit-rate codec, suitable for robust voice communication over IP.

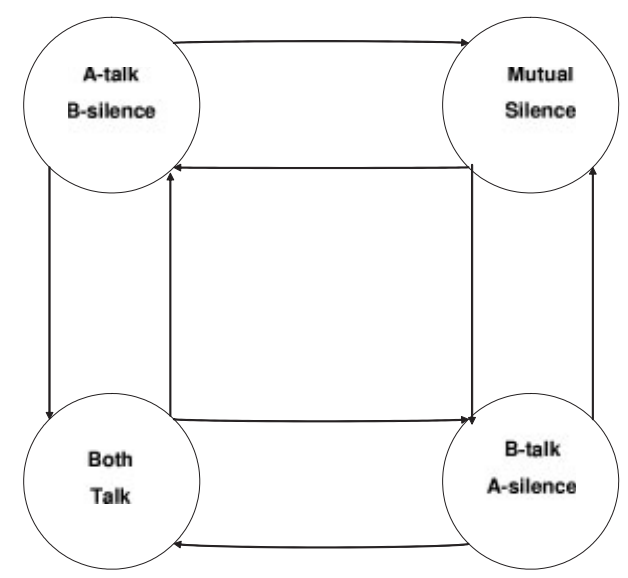

Figure 3. Conversational speech modelled as four-state Markov chain [14].

Different from the aforementioned models using a CBR voice traffic model, an On-Off traffic model, as specified in the ITU P.59 recommendation [13], was used in Reference [14] to analyse the network capacity of $802.11 \mathrm{a} / \mathrm{b} / \mathrm{g}$ WLANs, as shown in Figure 3 . The conversation between two users A and B is modelled as a four-state Markov chain, including (a) A talking B silent, (b) A silent B talking, (c) both talking and (d) both silent; the durations of states are mutually independent and identically distributed (i.i.d.) exponential random variables with means of 854 , 854,226 and $456 \mathrm{~ms}$, respectively. It is obtained that 22 and $102 \mathrm{G} .711$ connections can be accommodated in an $802.11 \mathrm{~b}$ and $802.11 \mathrm{a}$ WLAN, respectively. In other words, considering the silence intervals in a voice conversation, the voice capacity almost doubles compared to the results with a CBR traffic model.

In summary, for $802.11 \mathrm{~b}$ WLANs with very limited capacity, since all on-going voice traffic will be jeopardized if the AP becomes unstable, it is suggested to use a tight bound (the voice capacity obtained with the CBR traffic model) to limit the number of voice connections conservatively; on the other hand, when a WLAN can support a large number of voice connections, e.g. an $802.11 \mathrm{~g}$ WLAN, the On-Off traffic model can be used to fully explore the multiplexing gain.

In addition, the experimental and analytical results reveal that the voice capacity is a function of the system parameters, including transmission rate, voice packet payload length (dependent 
on the codec used), and packetization interval. Since the transmission rate is bounded according to the 802.11 standards, two feasible ways to increase the voice capacity are (a) either to enlarge the packetization interval (b) or to choose an efficient voice codec. Due to the large overheads at the MAC and physical layers, voice capacity is affected more by the packetization interval than by the payload length of voice packets. On the other hand, larger packetization interval brings more end-to-end delay, which may degrade the voice quality. There is a trade-off between the delay constraint and the voice capacity.

\subsection{Admission control}

A connection admission control (CAC) mechanism is to decide whether or not a new connection should be admitted into, and supported by, the system. The results in References [7,11] showed that placing an additional voice that exceeds the capacity of the WLAN will result in unacceptable quality for all ongoing VoIP connections. Therefore, admission control is necessary to maintain the quality for VoWLANs.

For infrastructure-based WLANs, admission control can be implemented at the AP, which can block traffic from any new connections when the current traffic load reaches the network capacity. If the WLAN supports voice traffic only, the voice capacity previously obtained can be applied directly for admission control. However, when the WLAN supports heterogeneous voice and data traffic, how to determine the appropriate admission region to efficiently utilize network resources and guarantee the voice quality is still an open issue.

Due to the difficulty in modelling the data traffic (for both long flows and short flows), and the randomness of the contention-based MAC protocol, most proposed CAC schemes for heterogeneous voice and data services in WLANs are measurement based [15-17]. For instance, in Reference [15], the channel utilization estimation (CUE) was used to perform CAC: if the total amount of current CUE and the CUE for the new voice connection is less than the maximum CUE, the new connection is admitted; if the data traffic can be curtailed, the restrictions for data traffic are calculated and enforced, and then the new voice connections is admitted; otherwise, the new voice connection is blocked.

The effectiveness of measurement-based admission control mechanism largely depends on the accuracy of measurement and the applied admission policies, and the measurement overhead is not negligible. To simplify the process of making admission decision, analysis-based admission control in WLANs requires further investigation.

On the other hand, since transmission control protocol (TCP)-controlled data traffic may aggressively probe for available bandwidth and introduce transient congestion to the network, the quality of voice traffic will be degraded in non-prioritized free contention WLANs. In addition to admission control, voice traffic needs further protection [18]. How to differentiate voice service from best effort data service by enhanced MAC protocol will be discussed in the following section.

\section{MAC LAYER QoS ENHANCEMENT}

VoIP quality is closely related to three factors: packet end-to-end delay, delay jitter (delay variation), and packet loss. A two-way conversation is very sensitive to delay and 
delay jitter, but it can tolerate some degree of packet losses, depending on the error-resilience of the codec used.

ITU has recommended that one-way end-to-end delay should be no greater than $150 \mathrm{~ms}$ for good voice quality, and up to $400 \mathrm{~ms}$ for acceptable voice quality, with an echo canceller [19]. The delay constraint is much more stringent when no echo canceller is adopted, and the end-toend delay should be limited to $25 \mathrm{~ms}$ for acceptable quality. For VoWLAN applications, delay includes codec delay, packetization delay, and network delay in both the WLAN and the backbone networks.

Delay jitter, or delay variation, mainly due to network dynamics, has even more negative effects on voice quality than that of delay. Since the WLAN is presumably the bottleneck, delay jitter in the WLAN is the dominant part. With the CBR traffic model for voice, delay jitter is mainly due to the random channel service time, the time duration the network interface has taken to successfully transmit a frame over the WLAN, which is determined by the MAC protocol and the data transmission rate. Delay jitter can be removed by adopting a small playout buffer at the receiver with an efficient playout algorithm [20]. Packets arriving at the receiver later than the playout time will be discarded. The playout buffer size should be carefully chosen; large playout buffer size may introduce additional delay, and small buffer size may result in considerable packet losses.

Packet loss is also a major source of impairment in VoIP systems. According to Shim et al. [21], a voice quality is considered acceptable only when the packet loss rate is less than $2 \%$. There are two sources of packet losses: (1) network packet losses, mainly due to network congestion (router buffer overflow), link failures and rerouting, transmission errors, etc.; and

(2) discarded packet losses for packets experienced excessive delay.

To support both voice and data applications over WLANs, it is important to design a MAC protocol with QoS support to voice traffic, implement an appropriate queue management scheme, develop efficient playout buffer algorithms, and increase the transmission rate with a more efficient physical layer protocol, etc. The playout buffer algorithms and physical layer design are out of the range of this paper. Here, we mainly focus on the QoS enhancement mechanisms in the MAC layer.

Whether the IEEE 802.11 MAC can provide desired QoS for voice connections has been extensively investigated. With the legacy 802.11 DCF mode, all stations, including the AP if available, compete for channel access with the same priority. There is no mechanism to assign higher priority to real-time traffic with stringent QoS requirements. In the MAC layer, two main approaches have been proposed to better support real-time applications: the polling-based mechanism and the prioritized contention-based mechanism.

\section{1. $P C F$}

The optional PCF mode is available in a centrally controlled WLAN, using a polling-based mechanism intended to guarantee delay for real-time applications. In Reference [22], the PCF performance has been studied via simulations and, it is concluded that an echo canceller is necessary since delay cannot be bounded within $25 \mathrm{~ms}$. The relationship between the network capacity and end-to-end delays of voice traffic has been obtained in Reference [23]. With larger inter-poll periods, more voice connections can be accommodated at the expense of increased delay. For example, 26 voice calls with codec rate of $8.5 \mathrm{kbps}$ can be accommodated if the interpoll period is $90 \mathrm{~ms}$ and the maximum delay is $303 \mathrm{~ms}$, whereas only 17 calls can be supported 


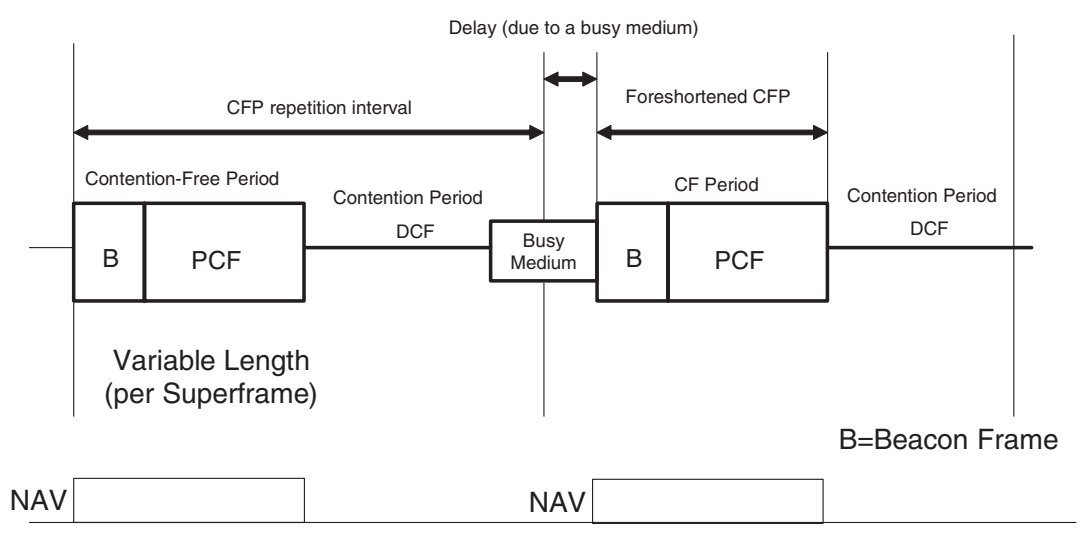

Figure 4. PCF stretch effect.

with the maximum delay of $213 \mathrm{~ms}$ if the inter-poll period is $60 \mathrm{~ms}$. Adaptive polling schemes have been proposed in Reference [24] to improve the bandwidth utilization by exploiting the voice traffic characteristics. Compared to the round-robin polling scheme, under high traffic load, the schemes proposed in Reference [24] can achieve better throughput and delay performance by introducing a talk spurt detection algorithm into the system.

PCF is not ideal for real-time traffic due to the following reasons [25]. First, the inefficient or complex centralized polling schemes cannot achieve desired performance when the traffic load increases [26]. There is always a trade-off between the efficiency and complexity of polling schemes: round-robin is simple but may introduce unnecessary polling overheads when the polled stations do not have data to transmit; by considering voice traffic characteristics, the polling scheme in Reference [24] is more efficient at the expense of more complexity. In addition, in the PCF mode, all communications have to go through the PC, which prolongs the delay in WLANs. Second, the incompatible co-operation between the DCF and PCF may cause so-called stretch effect, as shown in Figure 4; the contention period (CP) (with the DCF mode) may stretch to the CFP (with the PCF mode) if the last data frame starts transmission at the end of the CP, which leads to unpredictable delays in each CFP. Third, unknown transmission durations of polled stations may introduce uncontrolled delay for other stations. As discussed above, currently, PCF can only provide limited QoS for voice traffic, and thus it is not widely accepted as a good solution for VoWLAN systems.

\subsection{Differentiated schemes}

Service differentiation schemes have been proposed to provide better QoS for multimedia applications in IEEE 802.11 WLANs. Service differentiation can be achieved using priority queue management schemes and/or using different MAC parameters for different classes of traffic.

With priority queue schemes, traffic is classified into different priorities and each class of traffic occupies a separate queue. Within a station, packets buffered in a higher priority queue will be served earlier than those in a lower priority queue. A simple and viable prioritized contention-based approach to enhance the VoIP performance over 802.11 infrastructure-based 
WLAN has been presented in Reference [27] by implementing dual queues on the top of the 802.11 MAC controllers. The higher priority queue serves the real-time (RT) traffic, e.g. VoIP traffic, and the lower priority queue serves the non-real-time (NRT) traffic. The dual queues can be easily implemented in the driver of any 802.11 devices. In this way, only software upgrades are needed for 802.11 WLANs to provide certain level of QoS for real-time applications.

A similar but more complicated approach was proposed in Reference [28], which also used software upgrade-based approach with two separate queues for RT and NRT traffic. Instead of using the strict priority queue, it adopted the earliest deadline first (EDF)-scheduling algorithm for RT traffic, and an adaptive traffic smoother to regulate NRT traffic.

It has been demonstrated that the performance of VoIP can be enhanced significantly when VoIP traffic is separated from TCP traffic. The priority queue mechanism is well suitable for WLANs with only voice and data traffic. In a more complicated environment with heterogeneous traffic, such as video, voice, and data (interactive or non-interactive), how to extend the dual-queue approach and assign priorities to different traffic categories is a very challenging issue.

On the other hand, in the MAC layer, when multiple stations compete for transmission, higher priority traffic may use smaller contention window, slower backoff speed, or shorter IFS to obtain higher priority for transmission, and real-time traffic may allow less or no retransmission to reduce delay [28-35]. In Reference [29], for higher priority class traffic, the contention window is chosen from $\left[0,2^{j+1}-1\right]$, and for lower priority class traffic, it is chosen from $\left[2^{j+1}, 2^{j+2}-1\right]$, where $j$ is the backoff stage. In Reference [30], the inter-frame spaces (IFS) are differentiated to provide priority access. In Reference [31], priority schemes by differentiating the initial and maximum contention window have been proposed. In Reference [32], three priority schemes have been proposed: static priority scheduling (SPS), prioritized DIFS time mechanism, and prioritized backoff time distribution mechanism (PBTDM). Note that the scheme in Reference [29] is a special case of PBTDM. All these priority schemes have been evaluated by simulations. Analytical studies have also been reported in References [33-35].

However, these service differentiation schemes may not be operated well in heavy-load WLANs. For instance, smaller contention window sizes will result in more channel collisions due to contentions. As a result, higher priority frames may have even lower throughput and higher delay due to more collisions. This problem, referred as priority inversion problem, was demonstrated in References [36,37]. To address this problem, appropriate admission control schemes [38] or adaptive tuning of parameters have been proposed [39]. In conclusion, to support voice traffic over WLANs with QoS guarantee, both admission control and QoS-enhanced MAC are needed.

\section{3. $802.11 e$}

On the basis of differentiated schemes, the IEEE 802.11e standard, an enhancement of the legacy 802.11, is proposed to offer QoS support for multimedia applications [40,41].

First, the enhanced DCF (EDCF) is an extension of DCF with four levels of statistical access priority, enabling different traffic categories to be served in different priority queues, as shown in Figure 5. The contention-based channel access function of IEEE 802.11e, EDCF, adopts eight different priorities, which are further mapped into four access categories (AC). Access categories are achieved by differentiating the arbitration inter-frame space (AIFS), the initial contention window size, and the maximum window size. 


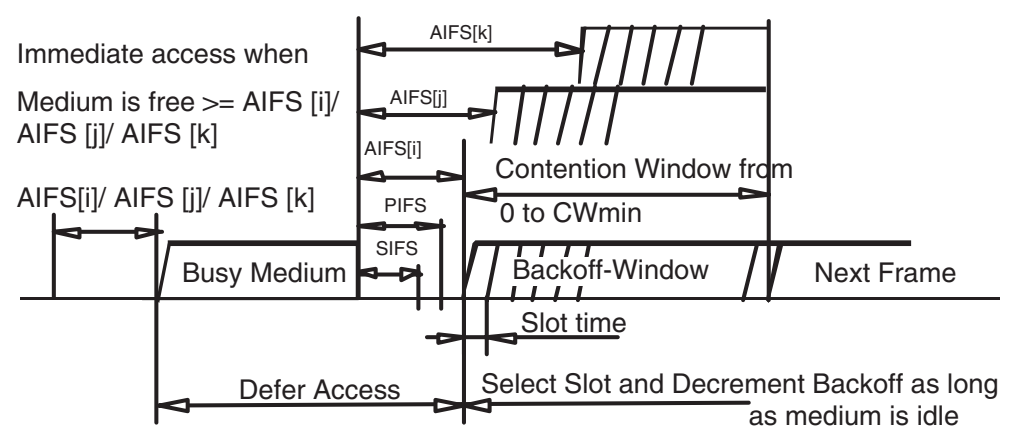

Figure 5. Enhanced distributed Co-ordination function.

With a smaller AIFS or window sizes, the higher priority class of traffic has a better chance to access the wireless medium. Although different priorities are implemented in EDCF, access to the medium is still determined according to the basic CSMA/CA mechanism. Therefore, EDCF is a prioritized contention-based mechanism.

Second, an extension of the PCF option called the hybrid co-ordination function (HCF) is proposed, which negotiates connections between an AP and the mobile stations, along with specifically assigned transmission durations for each frame. HCF also implements priority queue for different traffic categories so that voice traffic always has the highest priority than other traffic. Therefore, $\mathrm{HCF}$ is a hybrid of prioritized contention-based and polling-based mechanism for QoS provisioning. Unlike PCF, there is no specific boundary between the CP and CFP in 802.11e. The hybrid co-ordinator (HC) can poll any mobile station on the polling list in both CFP and CP, whereas the PC can only poll stations in CFP.

Third, the direct link protocol (DLC) permits two stations to communicate directly in the infrastructure-based WLAN, which significantly improve the network performance. Fourth, the group ACK mechanism, in which the receiver sends one ACK for a number of data packets received, can also reduce some overhead.

These new features of $802.11 \mathrm{e}$ can definitely improve the performance of voice traffic in a WLAN. However, there are still some concerns about the QoS guarantees that the protocol aims to provide. EDCF is a prioritized contention-based mechanism and cannot guarantee that low-priority packets will always wait until all packets with higher priority are transmitted. HCF suffers from the same complexity-efficiency trade-off in polling schemes as PCF does. Due to the complicated QoS provisioning mechanism specified in the protocol, it is very difficult to analyse the network performance of the 802.11e and to find the optimal parameters to achieve the best performance.

For EDCF, the analytical model for backoff-based priority scheme has been developed in Reference [33], which was further improved in References [35,42]. On the basis of Bianchi's saturated-station model [4], the analytical models for IFS-based priority scheme have been reported in References [43-46]. These models are developed under some assumptions to study protocol performance. However, the assumptions may not be realistic, e.g. saturated-station model or particular data traffic model, and thus the analytical results may not be applicable to real networks. To our best knowledge, no performance analysis of the HCF mode has been reported in the literature. Throughput and delay analysis via analytical models with more realistic assumptions in 802.11e-based WLAN is still an open issue. 
The implementation of the 802.11e protocol may be difficult and expensive since the upgrade or replacement of the existing WLANs is hardware based. Another issue is the compatibility. The 802.11e is designed to be backward compatible with the legacy 802.11 protocol. In 802.11e, AIFS is defined as an interval larger than or equal to DIFS. Therefore, the stations using EDCF may receive a lower priority than stations using DCF when they coexist in a WLAN, and this may discourage the deployment of IEEE 802.11e.

\section{VOICE OVER WLAN/CELLULAR SYSTEMS}

The cellular network has been well deployed to provide high-quality voice services for mobile users. It is anticipated that the WLAN is able to be integrated with the cellular network to provide VoIP services and Internet access anywhere, anytime. The WLAN/cellular systems, as shown in Figure 6, can take advantage of the wide coverage and almost universal roaming support of cellular networks and low-cost services in WLANs [47]. WLAN overlays extend the capacity of the cellular networks. How to maintain the voice connection and quality for mobile users in WLAN/cellular systems is a challenging issue.

To support seamless mobility, a large number of mobility management mechanisms in different layers have been proposed: the mobile IP protocol in the network layer [48], the end-toend approach by using dynamic updates to the domain name system (DNS) to track mobile host location [49], or the application layer SIP [50,51], etc. There is a rich body of literature that addresses mobility management issues in all-IP wireless systems, interested readers can refer to the survey paper [52]. In the following, we focus on two important issues for uninterrupted voice services: handoff and admission control.

\subsection{Horizontal and Vertical Handoffs}

As shown in Figure 6, a horizontal handoff occurs when a mobile user moves between the same type of networks, e.g. from WLAN1 to WLAN2. When a mobile user moves out of the coverage of a WLAN, and connects to the base station (BS) of a radio cell, or vice versa, the procedure is called a vertical handoff.

The procedure of horizontal handoff consists of three stages. First, the mobile user should make the handoff decision according to the received signal strength or other measurements. Second, the mobile user performs layer-2 handoff to another AP. Third, layer-3 handoff is performed to ensure the end-to-end connection if necessary. The layer- 2 handoff can further be separated into three steps. First, a mobile user tries to discover the APs by broadcasting probe request frames to collect information from all available APs, or directly collects information of APs from received beacon frames. This step accounts for the most part of the handoff delay. Then, the mobile user is authenticated by the new AP. Finally, the mobile users associate with the new AP and de-associate with the old AP if it receives the positive reply from the new AP. The horizontal handoff has been studied in Reference [53]. It is shown that the latency of horizontal handoff usually takes more than $150 \mathrm{~ms}$.

The vertical handoff is more complicated and takes longer time, depending on how tightly two wireless systems are coupled. In the tight coupling mode, WLANs are connected directly to the $3 \mathrm{G}$ core network, and traffic from WLANs goes through the core network to the BS, and vice versa. In the loose coupling mode, both the cellular network and WLANs are connected to 


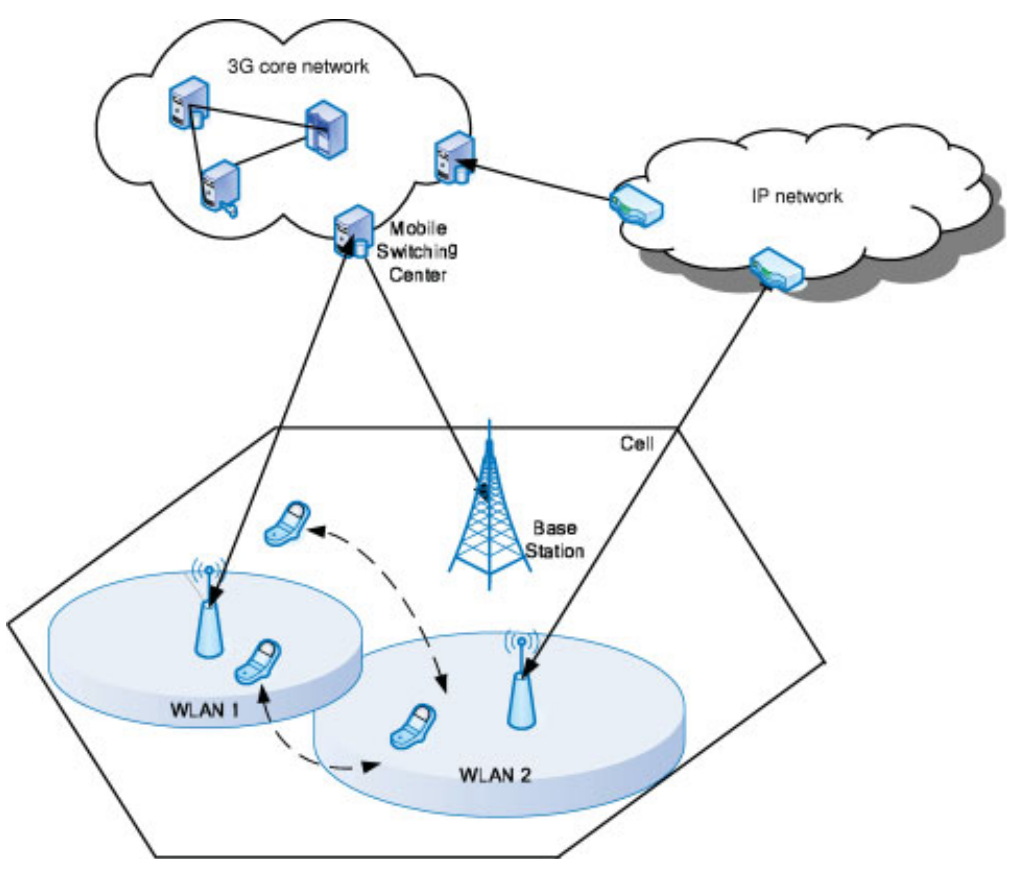

Figure 6. Integrated voice services over WLAN and cellular networks.

the Internet backbone, and data paths of the two networks are independent. As shown in Figure 6, WLAN1 is tightly coupled with the cellular network while WLAN 2 is loosely coupled. The vertical handoff between the loosely coupled General Packet Radio Service (GPRS) system and WLAN takes around $4 \mathrm{~s}$ (from WLAN to GPRS) or $7 \mathrm{~s}$ (from GPRS to WLAN) [54]. The vertical handoff between the third-generation $(3 \mathrm{G})$ cellular system and WLAN takes up to several hundred milliseconds $[55,56]$, which is still too lengthy to voice connections. Moreover, packets may be dropped by the old AP during the handoff procedure, which degrades, or even disconnects the voice connections during the handoff. Optimized fast handoff scheme has been proposed and analysed in the literature [57, 58]. The proposed scheme uses bicasting technique in which duplicated packets are sent to both air interfaces (cellular network and WLAN) for a short period during the handoff. It is shown in Reference [57] that fast handoff with bicasting technique can significantly reduce the handoff packet losses.

Currently, the $802.11 \mathrm{k}$ task group is working on the radio resource management to make efficient use of the WLAN resources. The APs can obtain the RF channel knowledge and make better roaming decisions according to the feedbacks from the mobile users, which can efficiently reduce the handoff latency. In this way, faster and uninterrupted wireless services can be provided. The $802.11 \mathrm{k}$ is now in the 'letter ballot' stage and it is unknown when the standard will be finished.

Meanwhile, the 802.11f task group specifies a protocol for the communications between multi-vendor APs, which is also known as the Inter-Access Point Protocol (IAPP). The 802.11f handles the interoperability of multi-vendor APs across a distribution system, and is necessary for fast handoff when a user is roaming among the coverage areas of multi-vendor APs. The 802.11f standard has been published in 2003 . 
On the other hand, the $802.11 \mathrm{r}$ task group is recently founded to address fast roaming among APs, although it is argued that the efforts in $802.11 \mathrm{f}, 802.11 \mathrm{k}$, and $802.11 \mathrm{i}^{\dagger \dagger}$ should be sufficient to solve the roaming problem, and thus $802.11 \mathrm{r}$ might not be necessary. Nevertheless, it is expected that by combining these protocols, including $802.11 \mathrm{i}, 802.11 \mathrm{k}, 802.11 \mathrm{f}$, and $802.11 \mathrm{r}$, a faster, securer, and seamless handoff of a voice connection between multiple WLANs or between the cellular network and WLANs can be achieved.

Besides the standardization activities in the 802.11 family, end-to-end approaches for seamless roaming and bandwidth aggregation are advocated in the literature, e.g. parallel TCP (pTCP) [59], stream control transmission protocol (SCTP) [60]. As the price of RF transceivers (radios) has fallen dramatically in recent years, the low cost allows multiple radios to be used in the same device. Using a multihomed transport layer protocol, multi-radio-equipped mobile hosts can establish multiple connections through both the WLAN and the cellular system simultaneously, which cannot only mitigate the negative effects during the handoff, but also achieve a higher data rate by aggregating the bandwidth in both wireless access networks.

\subsection{Admission control}

Measurement results showed that the performance of VoWLAN/cellular systems can degrade significantly with the increase of VoIP connections and user mobility [61]. Furthermore, the performance degradation affects all the existing VoIP connections, not just the new connection or the user which undergoes mobility. To guarantee the voice quality and efficiently utilize network resources of WLAN/cellular systems, admission control is necessary.

A general assignment strategy for admission control in wireless overlay networks have been proposed in Reference [62], which is not optimized for WLAN/Cellular interworking. Recently, an admission control strategy has been proposed for WLAN/Cellular systems [47], which considered the distinct characteristics of cellular systems and WLANs, the traffic characteristics of voice and data and their QoS requirements, and the user mobility pattern. With this strategy, the bandwidth of the cellular network can be saved for data traffic when some voice connections are admitted to the WLAN. As such, the traffic load can be effectively balanced between the two networks and the overall resource utilization is highly improved. It is anticipated more research will be conducted in the near future.

\section{CONCLUSION}

In this paper, we have presented an extensive survey on the voice capacity of an IEEE 802.11based WLAN and the QoS enhancement mechanisms in the MAC layer. Only a limited number of voice connections can be supported in an 802.11 WLAN because of the overhead and the inherent inefficiency of the MAC protocol. Accurate voice capacity estimation is critical for effective and efficient admission control for VoWLAN. When voice and data traffic share the wireless medium, it is important to design a MAC protocol with QoS support, implement appropriate queue management schemes, choose proper voice codecs, and develop efficient playout buffer algorithms to satisfy the stringent QoS requirements of voice traffic. We further study the handoff and admission control issues for voice over WLAN/cellular systems.

\footnotetext{
$\bar{\dagger}$ The IEEE $802.11 \mathrm{i}$ standard is intended to improve WLAN security.
} 
It is concluded that VoWLAN is a promising but very challenging technology that needs more efforts to achieve potential success in the future. Some important open issues beckon for further investigation: voice/data capacity analysis for effective admission control, voice performance analysis in QoS-enhanced 802.11e WLANs, admission control and optimal bandwidth aggregation in WLAN/cellular systems, network security and voice performance trade-off, etc.

\section{REFERENCES}

1. Schulzrinne H, Casner S, Frederick R, Jacobson V. RTP: a transport protocol for real-time applications. Available in ftp://ftp.ietf.org/rfc/rfc1889.txt, January 1996.

2. IEEE 802.11 WG. Part 11: Wireless LAN Medium Access Control (MAC) and Physical Layer (PHY) specification, August 1999.

3. Xiao Y, Rosdahl J. Throughput and delay limits of IEEE 802.11. IEEE Communications Letters 2002; 6(8):355-357.

4. Bianchi G. Performance analysis of the IEEE 802.11 distributed coordination function. IEEE JSAC 2000; 18(3):535-547.

5. Schwartz M. Broadband Integrated Networks. Prentice-Hall: New Jersey, 1996.

6. Anjum F et al. Voice performance in WLAN networks-an experimental study. Proceedings of the IEEE Globecom'03, vol. 6, San Francisco, CA, U.S.A., December 2003; 3504-3508.

7. Garg S, Kappes M. An experimental study of throughput for UDP and VoIP traffic in IEEE 802.11b networks. Proceedings of the IEEE WCNC'03, vol. 3, New Orleans, LA, U.S.A., March 2003; 1748-1753.

8. Patel T, Ogale V, Baek S, Cui N, Park R. Capacity estimation of VoIP channels on wireless networks. Available in http://www.ece.utexas.edu/〜 wireless/EE381K11_Spring03/projects/11.3.pdf, 2003.

9. Hole DP, Tobagi FA. Capacity of an IEEE $802.11 \mathrm{~b}$ wireless LAN supporting VoIP. Proceedings of the IEEE ICC'04, vol. 1, Madison, WI, U.S.A., June 2004; 196-201.

10. Garg S, Kappes M. Can I add a VoIP call? Proceedings of the IEEE ICC'03, vol. 2, Seattle, WA, U.S.A., May 2003; 779-783.

11. Cai L, Shen X, Mark JW, Cai L, Xiao Y. Voice capacity analysis of WLAN with imbalanced traffic. Proceedings of Qshine'05, Orlando, FL, U.S.A., August 2005.

12. Smavatkul N, Chen Y, Emeott S. Voice capacity evaluation of IEEE 802.11a with automatic rate selection. Proceedings of the IEEE Globecom'03, vol. 1, San Francisco, CA, U.S.A., December 2003; 518-522.

13. ITU. Artificial conversational speech, March 1993.

14. Medepalli K, Gopalakrishnan P, Kodama T. Voice capacity of IEEE $802.11 \mathrm{~b}, 802.11 \mathrm{a}$ and $802.11 \mathrm{~g}$ wireless LANs. Proceedings of the IEEE Globecom'04, vol. 3, Dallas, TX, U.S.A., November 2004; 1549-1553.

15. Garg S, Kappes M. Admission control for VoIP traffic in IEEE 802.11 networks. Proceedings of the IEEE Globecom'03, San Francisco, CA, U.S.A., December 2003; 3514-3518.

16. Xiao Y, Li H. Voice and video transmissions with global data parameter control for the IEEE 802.11e enhance distributed channel access. IEEE Transactions on Parallel and Distributed Systems 2004; 15(11):1041-1053.

17. Xiao Y, Li H. Local data control and admission control for ad hoc wireless networks. IEEE Transactions on Vehicular Technology 2004; 53(5):1558-1572.

18. Xiao Y, Li H, Choi S. Protection and guarantee for voice and video traffic in IEEE 802.11e wireless LANs. Proceedings of the IEEE Infocom'04. Hong Kong, China, March 2004; 2153-2163.

19. ITU-T. General characteristics of international telephone connections and international telephone circuits one-way transmission time, February 1996.

20. Pinto J, Christensen KJ. An algorithm for playout of packet voice based on adaptive adjustment of talkspurt silence periods. Proceedings of the IEEE 24th Conference on Local Computer Networks, 1999; 224-231.

21. Shim C, Xie L, Zhang B, Sloane C. How delay and packet loss impact voice quality in VoIP. Technical Report, Qovia, Inc., December 2003.

22. Crow BP, Widjaja I, Kim JG, Sakai PT. Investigation of the IEEE 802.11 medium access control (MAC) sublayer functions. Proceedings of the IEEE Infocom'97, vol. 13, Kobe, Japan, April 1997; 126-133.

23. Veeraraphavan M, Cocker N, Moors T. Support of voice services in IEEE 802.11 wireless LANs. Proceedings of the IEEE Infocom'01, vol. 1. Anchorage, Alaska, April 2001; 448-497.

24. Kim Y, Suh Y. Adaptive polling MAC schemes for IEEE 802.11 wireless LANs supporting voice-over-IP services. Wireless Communication and Mobile Computing 2004; 4:903-916.

25. Ni Q, Romdhani L, Turletti T. A survey of QoS enhancements for IEEE 802.11 wireless LAN. Wiley Journal of Wireless Communication and Mobile Computing (JWCMC) 2004; 4(5):547-566.

26. Lindgren A, Almquist A, Schelen O. Evaluation of quality of service schemes for IEEE 802.11 wireless LANs. Proceedings of the LCN'01, Colmar, France, November 2001. 
27. Yu J, Choi S, Lee J. Enhancement of VoIP over IEEE 802.11 WLAN via dual queue strategy. Proceedings of the IEEE ICC'04. Paris, France, June 2004; 3706-3711.

28. Jain A, Qiao D, Shin K. RT-WLAN: A soft real-time extension to the ORiNoco linux device driver. Proceedings of the IEEE PIMRC'03. Beijing, China, September 2003; 1434-1440.

29. Deng DJ, Chang RS. A priority scheme for IEEE 802.11 DCF access method. IEICE Transactions on Communications 1999; E82-B(1):96-102.

30. Aad I, Castelluccia C. Differentiation mechanisms for IEEE 802.11. Proceedings of IEEE Infocom'01, Anchorage, AK, U.S.A., 2001; 22-26.

31. Veres A, Campbell AT, Barry M, Sun L-H. Supporting service differentiation in wireless packet networks using distributed control. IEEE JSAC, Washigton, DC, U.S.A., 2001; 19(10):2081-2093.

32. Pallot X, Miller LE. Implementing message priority policies over an 802.11 based mobile ad hoc network. Proceedings of IEEE MILCOM'01, Washington, DC, U.S.A., 2001; 860-864.

33. Xiao Y. A simple and effective priority scheme for IEEE 802.11. IEEE Communications Letters 2003; 7(2): $70-72$.

34. Xiao Y, Pan Y. Differentiation, QoS Guarantee, and optimization for real-time traffic over one-hop ad hoc networks. IEEE Transactions on Parallel and Distributed Systems 2005; 16(65):538-549.

35. Xiao Y. Performance analysis of priority schemes for IEEE 802.11 and IEEE $802.11 \mathrm{e}$ wireless LANs. IEEE Transactions on Wireless Communications 2005; 4(4):1506-1515.

36. Ramos N, Panigrahi D, Dey S. Quality of service provisioning in 802.11 e networks: Challenges, approaches, and future directions. IEEE Network 2005; 19(4):14-20.

37. Malli M, Ni Q, Turletti T, Barakat C. Adaptive fair channel allocation for QoS enhancement in IEEE 802.11 wireless LANs. Proceedings of the IEEE ICC 2004, Madison, WI, U.S.A., June 2004; 3470-3475.

38. Gao D, Cai J, Ngan K. Admission control in IEEE 802.11e wireless LANs. IEEE Network 2005; 19(4):6-13.

39. Romdhani L, Ni Q, Turletti T. Adaptive EDCF: enhanced service differentiation for IEEE 802.11 wireless ad hoc networks. Proceedings of the IEEE WCNC'03, New Orleans, LA, U.S.A., March 2003; 1373-1378.

40. IEEE 802.11 WG. Wireless Medium Access Control (MAC) and Physical Layer (PHY) specifications: MAC enhancements for quality of service (QoS), July 2003.

41. Xiao Y. IEEE 802.11e: QoS provisioning at the MAC layer. IEEE Wireless Communication 2004; 11(3):72-79.

42. Xiao Y. An analysis for differentiated services in IEEE 802.11 and IEEE 802.11e wireless LANs. Proceedings of IEEE ICDCS'04, Tokyo, Japan, 2004; 32-39.

43. Xiao Y. Enhanced DCF of IEEE 802.11e to support QoS. Proceedings of IEEE WCNC 2003, New Orleans, LA, U.S.A., March 2003; 1291-1296.

44. Bianchi G, Tinnirello I. Analysis of priority mechanisms based on differentiated inter frame spacing in CSMA-CA. Proceedings of IEEE VTC'03, Orlando, FL, U.S.A., October 2003; 1401-1405.

45. Robinson JW, Randhawa TS. Saturation throughput analysis of IEEE 802.11e enhanced distributed coordination function. IEEE JSAC 2004; 22(5):535-547.

46. Kong Z, Tsang D, Bensaou B, Gao D. Performance analysis of IEEE 802.11e contention-based channel access. IEEE JSAC 2004; 22(10):2095-2106.

47. Song W, Jiang H, Zhuang W, Shen X. Resource management for QoS support in WLAN/cellular interworking. IEEE Network - special issue on 4G Network Technologies for Mobile Telecommunications, 2005; 19(5):12-18.

48. Perkins CE. IP mobility support for IPv4. RFC 3220, January 2002.

49. Snoeren AC, Balakrishnan H. An end-to-end approach to host mobility. Proceedings of the ACM MobiCom'00, Boston, MA, U.S.A., August 2000; 155-166.

50. Rosenberg J, Schulzrinne H, Camarillo G, Johnston A, Peterson J, Sparks R, Handley M, Schooler E. SIP:Session Initiation Protocol. IETF RFC 3261, June 2002.

51. Banerjee N, Das SK, Acharya A. SIP-based mobility architecture for next generation wireless networks. Proceedings of the IEEE PERCOM'05, 2005; 181-190.

52. Akyildiz I, Xie J, Mohanty S. A survey of mobility management in next-generation all-IP-based wireless systems. IEEE Wireless Communications Magazine 2004; 11(4):16-28.

53. Cheng SX. The intra-domain WLAN handoff for real-time applications and its implementation suggestion. Available in http://www.cs.sfu.ca/ $\sim$ jcliu/cmpt880/Report/wlan_hov_final_01.pdf.

54. Chakravorty R, Vidales P, Subramanian K, Pratt L, Crowcroft J. Performance issues with vertical handoversexperiences from GPRS cellular and WLAN hot-spots integration. Proceedings of the IEEE PERCOM'04, Orlando, FL, U.S.A., 2004.

55. Stemm M, Katz R. Vertical handoffs in wireless overlay networks. ACM Mobile Networks and Applications (MONET) 1998; 3(4):335-350.

56. Zhang W, Jaehnert J, Dolzer K. Design and evaluation of a handover decision strategy for 4th generation mobile networks. Proceedings of the IEEE VTC'03, vol. 3, Orlando, FL, U.S.A., April 2003; 1969-1973.

57. Shi M, Xu L, Shen X, Mark JW, Saleh A. Air interface switching and performance analysis for fast vertical handoff in cellular network and WLAN interworking. Wiley Journal of Wireless Communication and Mobile Computing $(J W C M C)$, to appear. 
58. Malki KE, Soliman H. Simultaneous bindings for mobile ipv6 fast handoffs. draft-elmalki-mobileip-bicastingv6-03, IETF Draft, October 2003.

59. Hsieh H, Sivakumar R. A transport layer approach for achieving aggregate bandwidths on multi-homed mobile hosts. Proceedings of the ACM MobiCom'02, Atlanta, GA, U.S.A., 2002; 83-94.

60. Ma L, Yu F, Leung V, Randhawa T. A new method to support UMTS/WLAN vertical handover using SCTP. Proceedings of the IEEE VTC'03, Orlando, FL, U.S.A., 2003; 44-51.

61. Rajavelsamy R, Jeedigunta V, Holur B, Choudhary M, Song O. Performance evaluation of VoIP over 3G-WLAN interworking system. IEEE WCNC'05, vol. 4, New Orleans, LA, U.S.A., 2005; 2312-2317.

62. Klein T, Han S. Assignment strategies for mobile data users in hierarchical overlay networks: performance of optimal and adaptive strategies. IEEE JSAC 2004; 22(5):849-861.

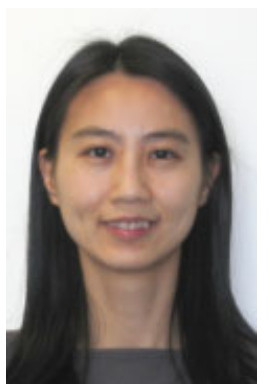

\section{AUTHORS’ BIOGRAPHIES}

Lin Cai received her BSc degree in Computer Science from Nanjing University of Science and Technology, Nanjing, China, in 1996 and MASc degree in Electrical and Computer Engineering from the University of Waterloo, Waterloo, Canada, in 2005. She is currently working toward a $\mathrm{PhD}$ degree in the same field at the University of Waterloo. Her current research interests include network performance analysis and protocol design for multimedia applications over IP-based wireless networks.

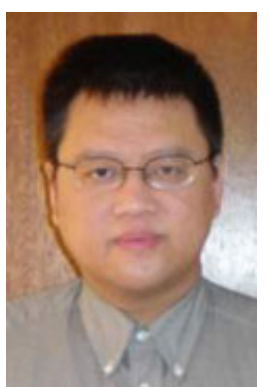

Yang Xiao (SM'04) worked at Micro Linear as a MAC (Medium Access Control) architect involving the IEEE 802.11 standard enhancement work before he joined the Department of Computer Science at The University of Memphis in 2002. Dr Xiao was a voting member of the IEEE 802.11 Working Group from 2001 to 2004. He is a senior member of the IEEE. He currently serves as Editor-in-Chief for International Journal of Security and Networks (IJSN) and for International Journal of Sensor Networks (IJSNet). He serves as an Associate Editor or on Editorial Boards for the following refereed journals: International Journal of Communication Systems (Wiley), Wireless Communications and Mobile Computing (Wiley), EURASIP Journal on Wireless Communications and Networking, and International Journal of Wireless and Mobile Computing. He has served as a lead/sole Journal Guest Editor for five journals during 2004-2005: International Journal of Security and Networks, EURASIP Journal on Wireless Communications and Networking, (Elsevier) Computer Communications Journal, (Wiley) Journal of Wireless Communications and Mobile Computing, and International Journal of Wireless and Mobile Computing. He serves as a referee for many funding agencies, as well as a panellist for the US NSF. His research areas include wireless networks and network security.

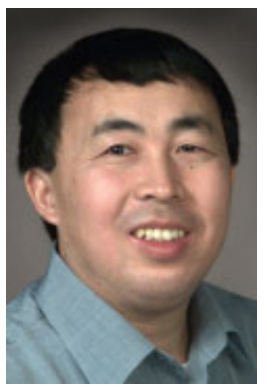

Xuemin (Sherman) Shen (M'97-SM'02) has been with the Department of Electrical and Computer Engineering, University of Waterloo, Canada, since October 1993, where he is a professor and the Associate Chair for Graduate Studies. His research focuses on mobility and resource management in interconnected wireless/wireline networks, UWB wireless communications systems, wireless security and ad hoc and sensor networks. He is a co-author of two books, and has published more than 200 papers and book chapters in wireless communications and networks, control, and filtering. He was Technical Co-Chair for the IEEE GLOBECOM'03, ISPAN'04, QShine'05 IEEE Broadnets'05 and WirelessCom'05, and is Special Track Chair of the 2005 IFIP Networking Conference. He serves as Associate Editor for IEEE Transactions on Wireless Communications, IEEE Transactions on Vehicular 
Technology, Computer Networks, ACM/Wireless Networks, Wireless Communications and Mobile Computing (Wiley), and International Journal Computer and applications. He has also served as Guest Editor for IEEE JSAC, IEEE Wireless Communications, and IEEE Communications Magazine. He received the Premier's Research Excellence Award (PREA) in 2003 from the Province of Ontario, Canada, for demonstrated excellence of scientific and academic contributions, and the Distinguished Performance Award in 2002 from the Faculty of Engineering, University of Waterloo, for outstanding contributions in teaching, scholarship, and service.

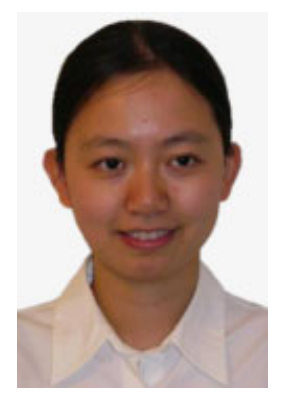

Lin Cai (M'06-S'00) received her MASc and $\mathrm{PhD}$ degrees (with Outstanding Achievement in Graduate Studies Award) in electrical and computer engineering from the University of Waterloo, Waterloo, Canada, in 2002 and 2005, respectively. Since July 2005, she has been an Assistant Professor in the Department of Electrical and Computer Engineering at the University of Victoria, Victoria, Canada. Her research interests span several areas in wireless communications and networking, with a focus on network protocol and architecture design supporting emerging multimedia traffic over wireless, mobile, and ad hoc and sensor networks. Her research work has been published in prestigious journals and conferences (e.g. IEEE Transactions on Mobile Computing, IEEE Transactions on Wireless Communications, IEEE Transactions on Multimedia, IEEE Transactions on Vehicular Technology, ACM MobiCom, IEEE Infocom). She serves as the Associate Editor for EURASIP Journal on Wireless Communications and Networking, and International Journal of Sensor Networks (IJSNet).

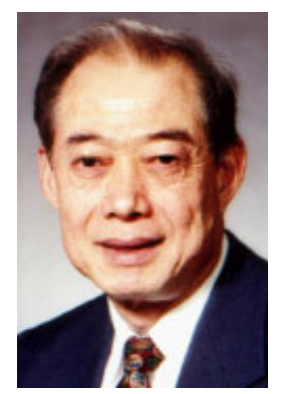

Jon W. Mark (LF) received his PhD degree in electrical engineering from McMaster University in 1970. In September 1970 he joined the Department of Electrical and Computer Engineering, University of Waterloo, Waterloo, Ontario, where he is currently a Distinguished Professor Emeritus. He served as the Department Chairman during July 1984-June 1990. In 1996 he established the Centre for Wireless Communications (CWC) at the University of Waterloo and is currently serving as its founding Director. Dr Mark had been on sabbatical leave at the following places: IBM Thomas J. Watson Research Center, Yorktown Heights, NY, as a Visiting Research Scientist (1976-1977); AT\&T Bell Laboratories, Murray Hill, NJ, as a Resident Consultant (1982-1983): Laboratoire MASI, Université Pierre et Marie Curie, Paris France, as an Invited Professor (1990-1991); and Department of Electrical Engineering, National University of Singapore, as a Visiting Professor (1994-1995).

He has previously worked in the areas of adaptive equalization, image and video coding, spread spectrum communications, computer communication networks, ATM switch design and traffic management. His current research interests are in broadband wireless communications, resource and mobility management, and cross domain interworking. He is a co-author of the text Wireless Communications and Networking, Prentice-Hall 2003. A Life Fellow of IEEE, Dr Mark is the recipient of the 2000 Canadian Award for Telecommunications Research and the 2000 Award of Merit of the Education Foundation of the Federation of Chinese Canadian Professionals. He was an editor of IEEE Transactions on Communications (1983-1990), a member of the Inter-Society Steering Committee of the IEEE/ACM Transactions on Networking (1992-2003), a member of the IEEE Communications Society Awards Committee (1995-1998), an editor of Wireless Networks (1993-2004), and an associate editor of Telecommunication Systems (1994-2004). 